\title{
Letter
}

\section{Acute hepatotoxicity induced by quetiapine fumarate in larval zebrafish}

\author{
Jinfeng Liang ${ }^{1, *}$, Wangdong Jin', \\ Hongcui Liư ${ }^{5}$, Yanfeng Huang ${ }^{6}$, Chunqi Li5,6, Li Zhou² and Thomas Efferth ${ }^{7}$ \\ 'Zhejiang Center for Drugs and Cosmetics Evaluation, Zhejiang Province Food and Drug Administration, \\ Hangzhou 310012, China \\ 2Institute of Orthopaedics and Traumatology, Zhejiang Chinese Medical University, Hangzhou 310053, China \\ ${ }^{3}$ The Biospecimen Repository, Zhejiang Cancer Hospital, Hangzhou 310022, China \\ ${ }^{4}$ Zhejiang Pharmaceutical College, Ningbo 315100, China \\ ${ }^{5}$ Hunter Biotechnology, Inc., Transfarland, Hangzhou 310012, China \\ ${ }^{6}$ Wenzhou Medical University, Wenzhou 325035, China \\ 'Department of Pharmaceutical Biology, Institute of Pharmacy and Biochemistry, Johannes Gutenberg University, \\ Mainz 55128, Germany
}

(Received April 18, 2016; Accepted April 27, 2016)

\begin{abstract}
Quetiapine fumarate $(\mathrm{QF})$ is a widely used antipsychotic agent for the first-line treatment of schizophrenia, with good tolerability and compliance in humans and no observed adverse effects against liver. Taking advantages of zebrafish, which can be utilized in rapid drug screening and acute toxicity assessment, our study determined a certain hepatotoxicity of QF for the first time, indicating a potential safety hazard of this commonly used drug to humans.
\end{abstract}

Key words: Quetiapine fumarate, Larval zebrafish, Hepatotoxicity, Liver, Yolk sac

\section{INTRODUCTION}

The liver is a major target of drug-induced toxicity or injury owing to the crucial role of this organ in drug biotransformation and metabolism processes. Drug-induced hepatotoxicity (DIHT) or liver injury is now recognized as a significant primary cause of acute/chronic liver diseases and the subsequent deaths in Western countries, such as fulminant hepatic failure leading to jaundice, coagulopathy, and multisystem organ failures with an estimated 3.5 deaths per million people in the US (Lee, 2003; Khashab et al., 2007; Hoofnagle et al., 1995). The incidence of such liver disorders has been estimated as $\sim 19$ new cases per 100,000 persons each year and accounts for 11 and $32 \%$ of all acute liver failure cases in the US and Europe (Bjornsson et al., 2013; Reuben et al., 2010; Hadem et al., 2012). DIHT remains the most common reason of drug failure in clinical trials and of post-market warnings and withdrawals of approved drugs (Norris et al., 2008; MacDonald and Robertson, 2009). The hepatotoxic drugs or secondary metabolites may exert toxic effects on critical organelles (e.g. mitochondria) in the hepatocytes or on drug transporters (e.g. P-glycoprotein), by changing structures of liver proteins, depleting glutathione (GSH) and inducing lipid peroxidation (Guengerich and Shimada, 1991; Jollow et al., 1973). The consequences are hepatocellular necrosis, apoptosis and sensitization to cytokine or inflammatory mediators. Currently, DIHT occurrence is attributable to the failed predictability of preclinical assays using traditional in vitro and in vivo models. The in vitro models fail to recapitulate the complexity of the intact organism and have less than $25 \%$ sensitivity for detection of hepatotoxins, whereas in vivo models are very laborious, low throughput, costly and time-consuming (Eckardt et al., 1998; O'Brien et al., 2006; O‘Brien et al., 2003). Accordingly, new models which can improve our ability to identify the DIHT earlier in drug development process are urgently needed. In the past few years, zebrafish has attracted increasing attention as a promising tool for such modeling purpose (Milan et al., 2003).

Zebrafish (Danio rerio), a cyprinid teleost, is emerging as a predictive vertebrate model animal used for toxicity assay of drugs (Selderslaghs et al., 2012). The mor-

Correspondence: Li Zhou (E-mail: zhouli0117@foxmail.com)

*These authors equally contributed to this work. 


\section{J. Liang et al.}

phological and molecular basis of tissues and organs in zebrafish is either identical or similar to humans, possessing orthologs for $\sim 85 \%$ of human genes and $>86 \%$ of human drug targets (Chen and Fishman, 1996; Granato and Nüsslein-Volhard, 1996; Gunnarsson et al., 2008; Howe et al., 2013). It has been confirmed that zebrafish has a strikingly similar toxicity profile to mammalian and is more appropriate for identifying endpoints of toxicity and elucidating the toxicity mechanisms (McGrath and Li, 2008; Hill et al., 2012; Hill, 2005). Zebrafish complete primary liver morphogenesis by $48 \mathrm{hr}$ post-fertilization (hpf) and the liver is fully formed and function by $72 \mathrm{hpf}$. Physiologically, at $120 \mathrm{hpf}$, the liver functions of larval zebrafish, including bile formation and excretion, serum protein secretion, digestion, metabolism, and storage of nutrients, synthesis of enzymes and other cofactors, lipogenesis, and xenobiotic metabolism are fully operational, are the same as that of mammals (Chu and Sadler, 2009). Most cell types in human liver are present in the larval zebrafish liver, and the latter has analogous mechanisms for handling xenobiotic compounds as the former, including both phase 1 and phase 2 biotransformation (Chu and Sadler, 2009; Alderton et al., 2010; Jones et al., 2010). Morphologically, massive hepatocellular necrosis can result in smaller, dark livers owing to the loss of transparency and these have been suggested as a phenotypic endpoint for assessing hepatotoxicity (Hill et al., 2012). When exposed to a hepatotoxicant, changes to liver morphology can be evaluated visually since larval zebrafish is virtually transparent (Hill et al., 2012; He et $a l ., 2013$ ). The unique nature of larval zebrafish presented above makes this animal an attractive model to study DIHT, being capable of mimicking the complexity of the whole liver system in humans.

Quetiapine fumarate (Seroquel $\AA$; AstraZeneca, London, $\mathrm{UK}$ ) is a second-generation antipsychotic agent of the dibenzothiazepine class used for control of positive and negative symptoms in patients with refractory psychosis, mood disorders, and bipolar disorders (Lacy et al., 2001). It was firstly approved by the FDA in 1997 for the treatment of schizophrenia, and recently approved in 2004 as a monotherapy and adjunct therapy with lithium or divalproex for the short-term treatment of acute manic episodes associated with bipolar I disorder. The molecular formula is $\mathrm{C}_{46} \mathrm{H}_{54} \mathrm{~N}_{6} \mathrm{O}_{8} \mathrm{~S}_{2}$ with a molecular weight of 883.1. The chemical designation is 2 [2-(4-dibenzo [b,f] thiazepin-11-yl-1-piperazinyl)ethoxy]-ethanol fumarate (2:1) salt (Lee, 2003; Bjornsson et al., 2013), as shown in Fig. 1. Quetiapine fumarate (QF) exerts its effect through a combination of dopamine type $2\left(\mathrm{D}_{2}\right)$ and 5-hydroxytryptamine-2 (5HT2) receptor antagonism. It also has affinities to a range of other neurotransmitter receptors such as serotonin $5 \mathrm{HT}_{1 \mathrm{a}}$, D1, histamine $\mathrm{H} 1$, and adrenergic $\alpha-1$ and $\alpha-2$, other than to cholinergic muscarinic and benzodiazepine receptors (McConville et al., 2000). As proposed for the first-line and long-term treatment, QF provides superior efficacy with better patient compliance and fewer side effects, and has been particularly appropriate in patients at the beginning of their illness (Green, 1999). It could generally be well tolerated by the elders and has long been deemed safer than other antipsychotic agents (Hustey and Duffull, 1999). To date, QF has rarely been linked to DIHT by experimental reports. In this study, via the transparent model of larval zebrafish, QF was assessed for evaluating its in vivo acute hepatotoxicity for the first time.

\section{MATERIALS AND METHODS}

\section{Drug preparation}

Commercial tablet of QF (batch number: B141137) was purchased from Hunan Dongting Pharmaceutical Co., Ltd. (Changde, China) and stored in room temperature. One tablet with an additive $(0.1 \mathrm{~g})$ was powdered and dissolved with ultra-pure water into a $10 \mathrm{mg} / \mathrm{mL}$ stock solution for use.

\section{Zebrafish handling}

A wild-type AB strain of zebrafish (batch number: 20150319) was employed for this study. Four to five pairs of adult zebrafish were randomly set up for each mating in a temperature- and light- controlled aquaculture facility with a standard 14:10 hr day/night photoperiod and fed with live brine shrimp twice a day and dry flake once a day. About 200-300 embryos were then generated and maintained at $28^{\circ} \mathrm{C}$ in fish water $(0.2 \%$ Instant Ocean Salt in deionized water, $\mathrm{pH} 6.9-7.2$, conductivity $480-510 \mu \mathrm{S} /$ $\mathrm{cm}$, and hardness 53.7-71.6 $\mathrm{mg} / \mathrm{L} \mathrm{CaCO}_{3}$ ). The embryos were washed and staged at 6 and $24 \mathrm{hpf}$ to obtain larval zebrafish. The zebrafish facility at Hunter Biotechnology, Inc is accredited by the Association for Assessment and

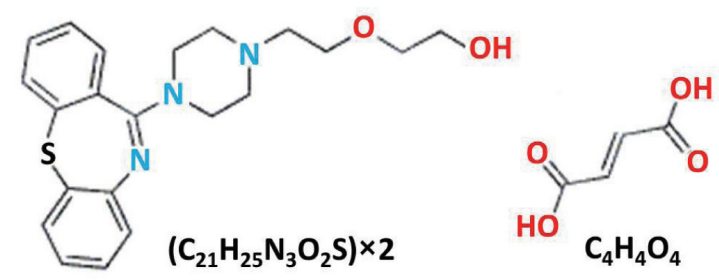

Fig. 1. Chemical structure of $Q F,\left(C_{21} \mathrm{H}_{25} \mathrm{~N}_{3} \mathrm{O}_{2} \mathrm{~S}\right)_{2} \mathrm{C}_{4} \mathrm{H}_{4} \mathrm{O}_{4}$. 
Quetiapine fumarate induces acute hepatotoxicity

Accreditation of Laboratory Animal Care (AAALAC) International.

\section{Drug treatment}

Larval zebrafish (72 hdf) were grouped ( $\mathrm{n}=30$ per group) and distributed into 6-well plates (Nest Biotech, China) in $3 \mathrm{~mL}$ fresh fish water for a treatment period of $48 \mathrm{hr}$ until $120 \mathrm{hpf}$. QF solution was dissolved in fish water to mimic oral administration. Untreated larvae was used as blank control. The dissolved oxygen concentration in each well was maintained above $80 \%$ throughout the experiments. After $48 \mathrm{hr}$ treatment of QF, larval zebrafish were subject to visual observation and image acquisition under a dissecting stereomicroscope (Olympus, Japan).

\section{$\mathrm{LC}_{10}$, NOAEL, and LOAEL and estimation}

Larval zebrafish was treated with QF from 72 to $120 \mathrm{hpf}$. Fish mortality was recorded every $24 \mathrm{hr}$, and dead zebrafish were counted as its heartbeat stopped under the dissecting stereomicroscope (Nikon, Japan). According to the preliminary experiments, ten concentrations $(0$, $20,30,35,40,45,50,55,60,65 \mu \mathrm{g} / \mathrm{mL})$ were selected to produce a mortality curve via Origin 8.0 (OriginLab, USA). The $\mathrm{LC}_{10}$ (10\% lethal concentration) was determined with logistic regression. Meanwhile, toxic manifestations in every larval were observed in double-blind to estimate the NOAEL (no observed adverse effect level) and LOAEL (lowest observed adverse effect level).

\section{Hepatotoxicity assessment}

Four concentrations $\left(1 / 10 \mathrm{LC}_{10}, 1 / 3 \mathrm{LC}_{10}, \mathrm{LC}_{10}\right.$ and 4/3 $\mathrm{LC}_{10}$ ) of $\mathrm{QF}$ were selected for hepatotoxicity assessment. With $48 \mathrm{hr}$ treatment from 72 to $120 \mathrm{hpf}$, the larval were visually observed for imaging acquisition of specific phenotypic endpoints under the dissecting stereomicroscope installed with a high-speed video camera. As described by $\mathrm{He}$ et al., the larval liver is approximately globular in structure with a clearly recognizable periphery against the neighboring tissues and is perfused with circulating blood cells (He et al., 2013). The normal liver is clear, whereas the hepatotoxicity attacked liver becomes darker with a brown or gray coloration and the texture of liver tissue becomes amorphous undergoing degeneration and/or necrosis (McGrath and Li, 2008; Hill et al., 2012). Three hepatotoxic phenotypic endpoints (liver size changes, liver degeneration, and yolk absorption delay) were adopted for assessing hepatotoxicity and calculated based on the formulas below:

Liver size (\%)

$=$ liver area $($ treated $) /$ liver area $($ control $) \times 100 \%$
Liver degeneration (\%)

$=[$ liver optical density (treated) - liver optical density (control)] / liver optical density (control) $\times 100 \%$

Yolk absorption delay (\%)

$=[$ yolk sac area (treated) - yolk sac area $($ control $)] /$ yolk sac area (control) $\times 100 \%$

Phenotypic quantitative analyses on liver size and opacity as well as yolk sac size were conducted by using image-based morphometric analysis (NIS-Elements D3.1, Japan).

\section{Statistical analysis}

All tests were replicated until the experimental condition was optimized. Sigmoidal regression for concentration-response curve was used for estimation of $\mathrm{LC}_{10}$ in Origin 8.0. One-way ANOVA followed by Dunnett's test was used to compare differences between different groups. All statistical analyses were performed using SPSS 16.0 software (SPSS Inc., Chicago, USA) and $P<0.05$ was considered statistically significant. For the quantitative hepatotoxicity analyses, all data were presented as mean \pm standard error (S.E.).

\section{RESULTS}

\section{LC $_{10}$, NOAEL, and LOAEL determination}

A dosage-mortality curve of larval zebrafish was presented in Fig. 2. QF induced death was observed in a dose-dependent manner, and no survival could be seen with QF above $55.0 \mu \mathrm{g} / \mathrm{mL}$ and no death occurred with QF below $20 \mu \mathrm{g} / \mathrm{mL}$. By means of sigmoidal regression, $\mathrm{LC}_{10}$ of QF was estimated as $39.0 \mu \mathrm{g} / \mathrm{mL}$. Through the preliminary replications and double blind observations, the NOAEL and LOAEL could be estimated as $3.9 \mu \mathrm{g} / \mathrm{mL}\left(1 / 10 \mathrm{LC}_{10}\right)$ and $13.0 \mu \mathrm{g} / \mathrm{mL}\left(1 / 3 \mathrm{LC}_{10}\right)$, respectively.

\section{Qualitative assessment of hepatotoxicity}

As shown in Fig. 3, hepatotoxicity-associated manifestations in larval zebrafish were observed. The untreated larvae exhibited clear liver tissue perfused with circulating blood cells. The larvae livers were found turned to non-transparent from brown to dark and liver blood flow was hardly observable with QF treatment at doses from 13.0 to $52.0 \mu \mathrm{g} / \mathrm{mL}$ in a dose-dependent manner. Neither hepatomegaly nor hepatoatrophy was observed in the QF treated larvae as compared with the control. A grossly swollen yolk sac was only seen in each larvae treated with QF at $13.0 \mu \mathrm{g} / \mathrm{mL}$ and above doses, indicating an observably delay of yolk absorption. The occurrence rate of QF-induced hepatotoxicity in zebrafish attained 100\% (30/30). 


\section{Quantitative assessment of hepatotoxicity}

The QF-induced changes of liver size, liver opacity, and yolk sac size in larval zebrafish relative to the normal control were further measured using an image-based

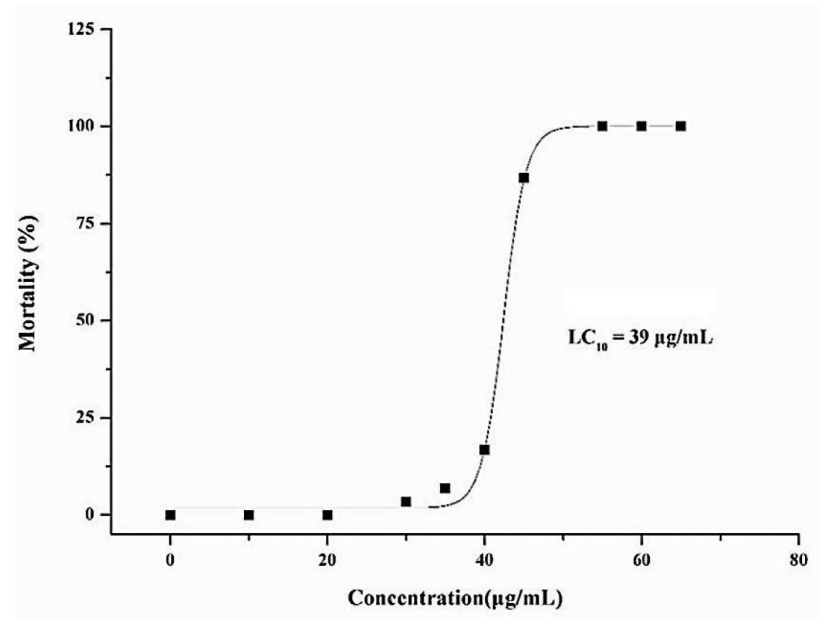

Fig. 2. QF-induced dose-dependent mortality of larval zebrafish between 72 and $120 \mathrm{hpf}(\mathrm{n}=30)$. morphometric analysis. Little change of liver size was seen between the control and each of the treated groups (Fig. 4), whereas significant increases of liver opacity and yolk sac size presented with QF treatment at doses from 13.0 to $52.0 \mu \mathrm{g} / \mathrm{mL}$, indicating a severe liver degeneration and yolk absorption delay induced by QF (Figs. 5,6). Such hepatotoxicity was exerted in a dosedependent manner. Furthermore, a dramatic change was found induced by QF at $\mathrm{LC}_{10}(39 \mu \mathrm{g} / \mathrm{mL})$, in which yolk sac size was 4.4-fold increased and continued to get 5.0fold increased at QF dose above $\mathrm{LC}_{10}(52 \mu \mathrm{g} / \mathrm{mL})$.

\section{DISCUSSION}

As a first-line antipsychotics for management of schizophrenia, QF has good tolerability and compliance in humans with lowest incidence of adverse events among all antipsychotic agents (Hustey and Duffull, 1999; Cheer and Wagstaff, 2004). Unfortunately, QF abuse (overdose) has become more common in clinical cases, resulting in a variety of adverse reactions including cardiovascular disorders (e.g. palpitation, tachycardia, agitation, anxiety, hypotension), gastrointestinal disorders (e.g abdomi-
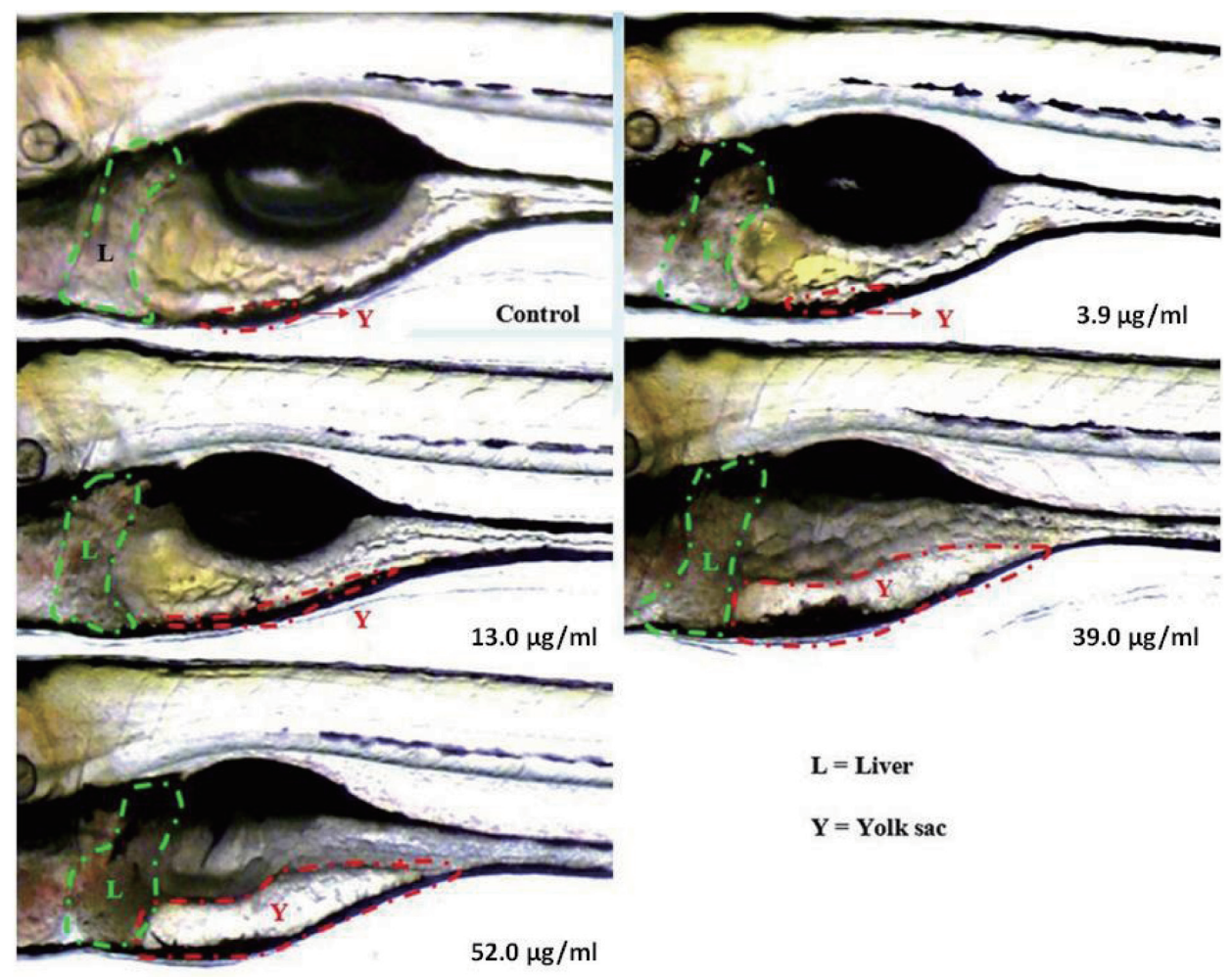

$$
\begin{aligned}
& \mathbf{L}=\text { Liver } \\
& \mathbf{Y}=\text { Yolk sac }
\end{aligned}
$$

Fig. 3. Observation of larval zebrafish at $120 \mathrm{hpf}$ after treatment of QF for $48 \mathrm{hr}$. The circled L and Y were larvae liver and yolk sac. 
Quetiapine fumarate induces acute hepatotoxicity

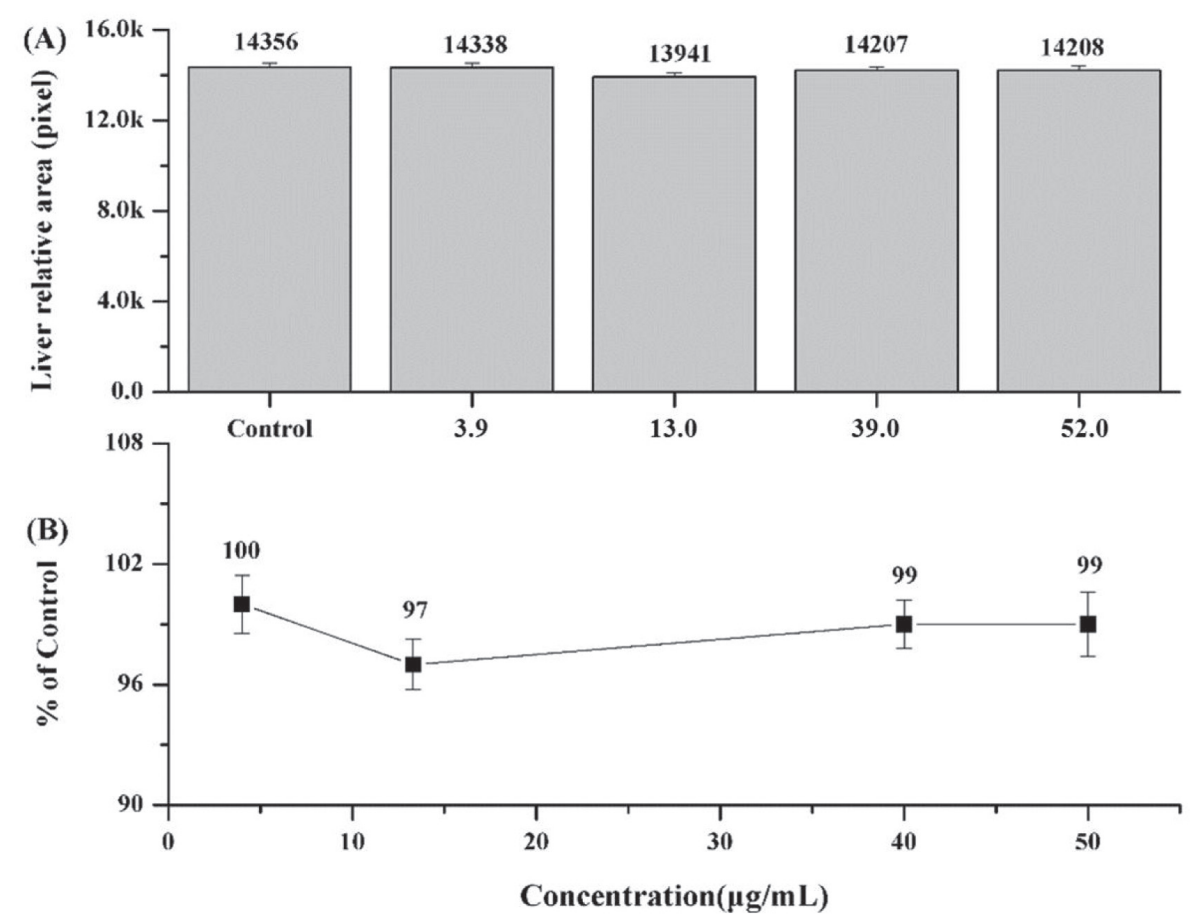

Fig. 4. Liver size changes of larval zebrafish induced by QF. A: liver area; B: liver area relative to control (\%). All data were represented as mean \pm standard error (S.E.).

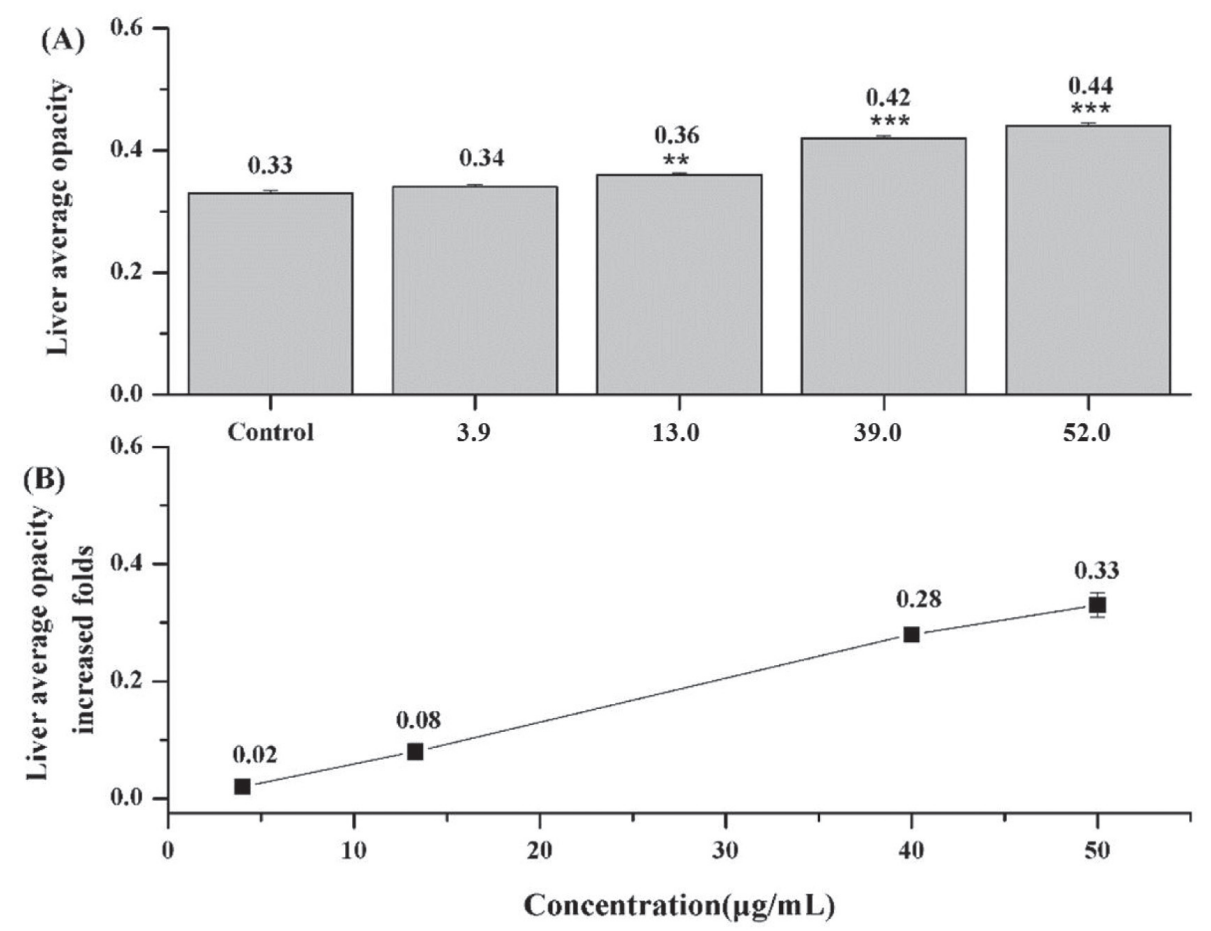

Fig. 5. Liver opacity changes of larval zebrafish induced by QF. A: liver opacity; B: increased folds of liver opacity to control (\%). All data were represented as mean \pm standard error (S.E.). $* * \mathrm{P}<0.01$ and $* * * \mathrm{P}<0.001$ vs. control. 


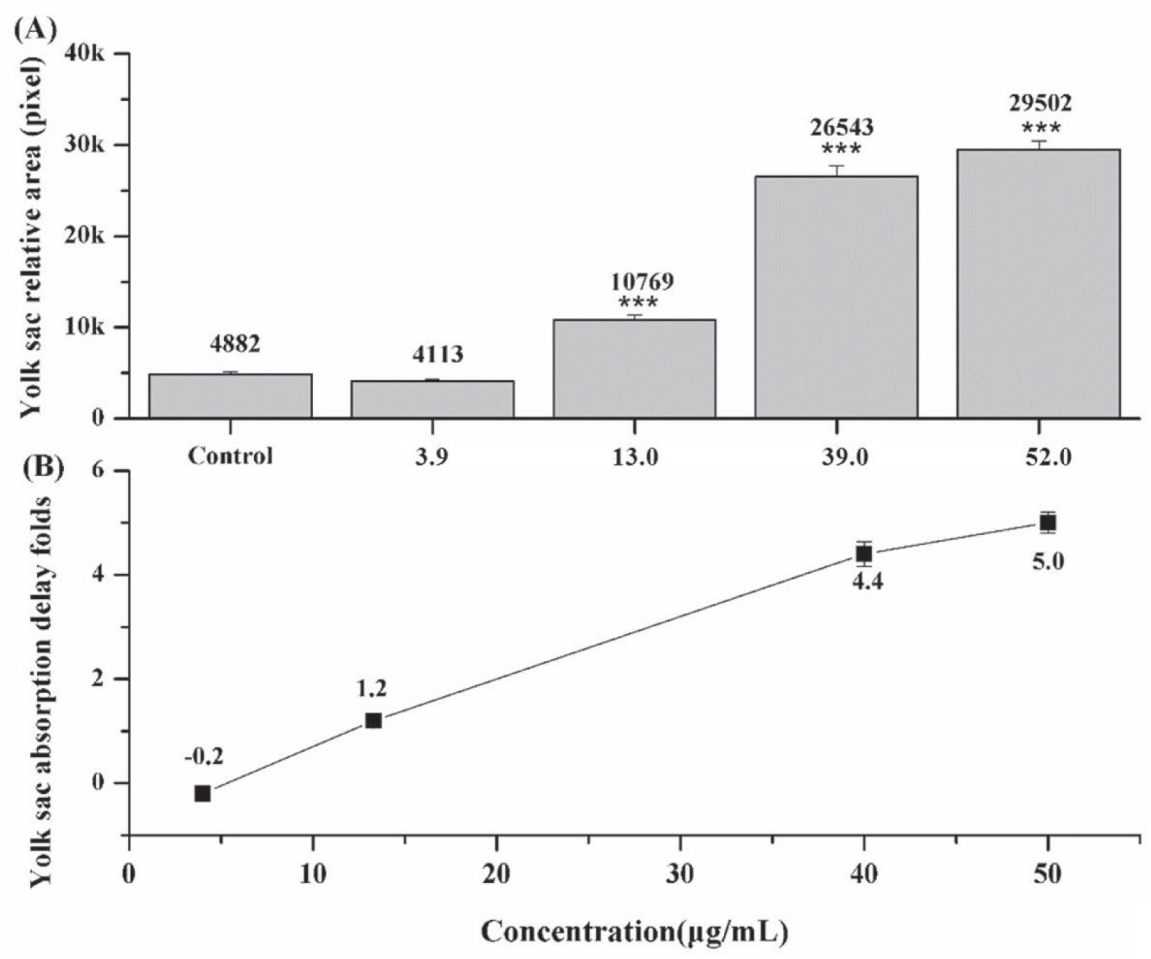

Fig. 6. Yolk sac size changes of larval zebrafish induced by QF. A: yolk sac area; B: increased folds of yolk sac area to control (\%). All data were represented as mean \pm standard error (S.E.). $* * \mathrm{P}<0.01$ and $* * * \mathrm{P}<0.001$ vs. control.

nal pain, dyspepsia, vomiting), and behavioral disorders (e.g tardive dyskinesia, myoclonus, dystonia, parkinsonism, akathisia, drowsiness, unconsciousness) (Rizos et al., 2009; Strachan and Benoff, 2006; Desarkar and Sinha, 2006; Bharadwaj and Grover, 2008; Shah et al., 2010; George et al., 2013; Dev and Raniwalla, 2000). The toxicity of QF is thought to be mediated by blockade of adrenergic $\alpha 1$ - and $\alpha 2$-receptors (hypotension and tachycardia) and blockade of histaminergic receptors (somnolence) (Pollak and Zbuk, 2000). Liver is the main site for QF metabolism and clearance, in which sulphoxidation took place by means of cytochrome P450 3A4 isoenzyme and QF extensively metabolized to over 20 metabolites and eliminated with urine ( $\sim 73 \%)$ and faeces ( $21 \%)$ (Green, 1999; Gunasekara and Spencer, 1998). Except to induce a mild and transient asymptomatic elevation of liver enzymes (AST, ALT, and bilirubin), QF was once considered to be a non-hepatotoxic agent by the market (Atasoy et al., 2007.). Afterwards to date, three clinical cases reported hepatic injury secondary to use of QF, raising a concern whether QF is of hepatotoxicity (Shpaner et al., 2008; Mutairi et al., 2012; El Hajj et al., 2004). However, it is insufficient to make a judgment on $\mathrm{QF}^{\text {'s }} \mathrm{s}$ hepa- totoxicity according to such extremely rare occurrences of liver abnormalities, since the etiology of liver injury could not be 100 percent determined and other concurrent medications (e.g. metformin and ramipril for diabetes and hypertension, respectively) might obscure the results in those ,case-by-case" reports (El Hajj et al., 2004; Ostapowicz et al., 2002). Therefore, reliable evidence is strongly needed for verifying the suspected hepatotoxicity of QF.

In the present study, a qualitative and quantitative in vivo assay using larval zebrafish was performed to determine the hepatotoxicity of QF. The morphological observation found that $\mathrm{QF}$ at $1 / 3 \mathrm{LC}_{10}(13.0 \mu \mathrm{g} / \mathrm{mL})$ induced typical toxic manifestations of liver (non-transparency) and yolk sac (edema), and the hepatotoxicity increased with increasing doses of QF (Fig. 3). The loss of liver transparency (being dark or brown) might be associated with reduction of liver blood flow, presenting a typical phenotype of liver degeneration that was also observed in other studies utilizing zebrafish to assess mammalian hepatotoxicants (Hill et al., 2012; Hill, 2011; Zhang et al., 2003). The edema of yolk sac was another hepatotoxic endpoint associated with an outcome of yolk 
Quetiapine fumarate induces acute hepatotoxicity

absorption delay in clinic. Correspondingly, the quantitative measurement showed a similar result - that is, both levels of liver opacity and yolk sac size were increased significantly with treatment of increasing doses of QF (Figs. 5, 6). The above hepatotoxic endpoints in larval zebrafish indicate a certain hepatotoxicity of QF, verifying the aforementioned deduction from the clinical ,caseby-case" reports. There was a significant positive correlation of lethality values and toxicity endpoints between zebrafish and rodents (mouse or rat), and a parallel comparison has shown that $\mathrm{LC}_{50}$ of cyclophosphamide in zebrafish $(650 \mu \mathrm{g} / \mathrm{mL})$ was about twice times higher than the $\mathrm{LC}_{50}(315 \mathrm{mg} / \mathrm{kg})$ in mouse (p.o.) (Zhang et al., 2003; Hill et al., 2008). Accordingly, in this study, the LOAEL $(13.0 \mu \mathrm{g} / \mathrm{mL}$ ) and above doses (upon to $52.0 \mu \mathrm{g} /$ $\mathrm{mL})$ of QF can be converted as a potentially hepatotoxic dose range ( $\sim 6.5$ to $26.0 \mathrm{mg} / \mathrm{kg}$ ) to mouse (p.o.). By converting the clinical daily dose of QF from human (5.0 to $10.0 \mathrm{mg} / \mathrm{kg}$ ) to mouse ( 45.5 to $91.0 \mathrm{mg} / \mathrm{kg})$, it can be concluded that the $\mathrm{QF}^{\text {'s }} \mathrm{s}$ hepatotoxic doses (6.5 to 26.0 $\mathrm{mg} / \mathrm{kg}$ for mouse) determined by our assay are far more less than the minimal dose $(45.5 \mathrm{mg} / \mathrm{kg}$ for mouse) applied in clinic, indicating a hepatotoxicity associated potential safety hazard to patients exposed to QF. Covering our findings, the overall prediction success rate of zebrafish for toxicity of drugs attained $100 \%$ and can thereby be ranked as excellent $(>85 \%)$ for identifying toxicity agents by the ECVAM (European Center for the Validation of Alternative Methods) criteria (Genschow et al., 2002).

A question is, why the traditional assays using cell lines or rodent animals could not predict the hepatotoxicity of QF during its development process, but zebrafish can? It may be due to the advantages of larval zebrafish, such as transparent body and hypersensitive response to toxicity, so that the potential or chronic organ toxicity could not be easily ignored. The transparency can achieve rapid and specific observation on morphologies and functions of liver and yolk sac, and the sensitivity of larval zebrafish can maximize the potential toxic responses to drugs that may not be seen in rodents (McGrath and Li, 2008). Other studies have also verified the zebrafish as a highly predictive animal model for in vivo evaluation of drug hepatotoxicity (He et al., 2013; Du et al., 2009; Mesens et al., 2015). Using zebrafish as an alternative animal model for drug screening can not only greatly accelerate the drug discovery process, decrease costs, and provide more specific results than traditional assays, but can also early predict the drug toxicity before drug development, and more importantly, determine the potential toxic hazard of commonly used drugs in clinic, such as QF. This study pro- vides the first experimental evidence for $\mathrm{QF}^{\text {' }} \mathrm{s}$ hepatotoxicity, suggesting that a particular consideration should be given to the QF-dependent patients for their liver functions and physicians should be aware of the potential serious hepatotoxicity of QF prior to its use. There is clearly a need to further improve insights into the molecular mechanisms of QF's hepatotoxicity in vivo.

\section{ACKNOWLEDGMENTS}

This work was supported by the Science and Technology Project Grant of Zhejiang Food and Drug Administration system (Grant no. 2014002), National Natural Science Foundation of China (Grant no. 81302989), Zhejiang Provincial Science and Technology Project of Traditional Chinese Medicine of China (Grant no. 2013ZQ007), Independent Project of Zhejiang Provincial Scientific and Technological Innovation Team of China (Grant no. 2011R50022-02), Research Fund for the Doctoral Program of Higher Education of China (Grant no. 20133322120006), Scientific research project of Zhejiang Provincial Department of Education (Grant no. Y201534534, Y201226186), and Ningbo Natural Science Foundation (Grant no. 2014A610277).

Conflict of interest---- The authors declare that there is no conflict of interest.

\section{REFERENCES}

Alderton, W., Berghmans, S., Butler, P., Chassaing, H., Fleming, A., Golder, Z., et al. (2010): Accumulation and metabolism of drugs and CYP probe substrates in zebrafish larvae. Xenobiotica, 40, 547-557.

Atasoy, N., Erdogan, A., Yalug, I., Ozturk, U., Konuk, N., Atik, L. and Ustundag, Y. (2007): A review of liver function tests during treatment with atypical antipsychotic drugs: a chart review study. Prog. Neuropsychopharmacol. Biol. Psychiatry, 31, 1255-1260.

Bharadwaj, R. and Grover, S. (2008): Parkinsonism and akathisia with quetiapine: three case reports. J. Clin. Psychiatry, 69, 11891191.

Bjornsson, E.S., Bergmann, O.M., Bjornsson, H.K., et al. (2013): Incidence, presentation, and outcomes in patients with druginduced liver injury in the general population of Iceland. Gastroenterology, 144, 1419-1425.

Cheer, S.M. and Wagstaff, A.J. (2004): Quetiapine: a review of its use in the management of schizophrenia. CNS Drugs, 18, 173199.

Chen, J.N. and Fishman, M.C. (1996): Zebrafish tinman homolog demarcates the heart field and initiates myocardial differentiation. Development, 122, 3809-3816.

Chu, J. and Sadler, K.C. (2009): New school in liver development: lessons from zebrafish. Hepatology, 50, 1656-1663.

Desarkar, P. and Sinha, V.K. (2006): Quetiapine-induced acute dystonia and akathisia. Aust. N Z J. Psychiatry, 40, 607-608. 
Dev, V. and Raniwalla, J. (2000): Quetiapine: a review of its safety in the management of schizophrenia. Drug Saf., 23, 295-307.

Du, Y.B., Shi, X.J., Liu, C.S., Yu, K. and Zhou, B.S. (2009): Chronic effects of water-borne PFOS exposure on growth, survival and hepatotoxicity in zebrafish: A partial life-cycle test. Chemosphere, 74, 723-729.

Eckardt, L., Haverkamp, W., Borggrefe, M. and Breithardt, G. (1998): Experimental models of torsade de pointes. Cardiovasc. Res., 39, 178-193.

El Hajj, I., Sharara, A.I. and Rockey, D.C. (2004): Subfulminant liver failure associated with quetiapine. Eur. J. Gastroenterol. Hepatol., 16, 1415-1418.

Genschow, E., Spielmann, H., Scholz, G., Seiler, A., Brown, N., Piersma, A., Brady, M., Clemann, N., Huuskonen, H., Paillard, F., Bremer, S. and Becker, K. (2002): The ECVAM international validation study on in vitro embryotoxicity tests: results of the definitive phase and evaluation of prediction models. European Centre for the Validation of Alternative Methods. Altern. Lab. Anim., 30, 151-176.

George, M., Haasz, M., Coronado, A., Salhanick, S., Korbel, L. and Kitzmiller, J.P. (2013): Acute dyskinesia, myoclonus, and akathisa in an adolescent male abusing quetiapine via nasal insufflations: a case study. BMC Pediatrics, 13, 187.

Granato, M. and Nüsslein-Volhard, C. (1996): Fishing for genes controlling development. Curr. Opin. Genet. Dev., 6, 461-468.

Green, B. (1999): Focus on quetiapine. Curr. Med. Res. Opin., 15, 145-151.

Guengerich, F.P. and Shimada, T. (1991): Oxidation of toxic and carcinogenic chemicals by human cytochrome P450 enzymes. Chem. Res. Toxicol., 4, 391-407.

Gunasekara, N.S. and Spencer, C.M. (1998): Quetiapine - a review of its use in schizophrenia. CNS Drugs, 9, 325-340.

Gunnarsson, L., Jauhiainen, A., Kristiansson, E., Nerman, O. and Larsson, D.G. (2008): Evolutionary conservation of human drug targets in organisms used for environmental risk assessments. Environ. Sci. Technol., 42, 5807-5813.

Hadem, J., Tacke, F., Bruns, T., et al. (2012): Etiologies and outcomes of acute liver failure in Germany. Clin. Gastroenterol. Hepatol., 10, 664-669.

He, J.H., Guo, S.Y., Zhu, F., Zhu, J.J., Chen, Y.X., Huang, C.J., Gao, J.M., Dong, Q.X., Xuan, Y.X. and Li, C.Q. (2013): A zebrafish phenotypic assay for assessing drug-induced hepatotoxicity. J. Pharmacol. Toxicol., 67, 25-32.

Hill, A., Ball, J., Jones, M., Dodd, A., Mesens, N. and Vanparys, P. (2008): Implementation of Zebrafish toxicity testing between in vitro and in vivo models to advance candidate selection. In Proceedings of the 29th Annual Meeting of the American College of Toxicology, Abstract \#108, Tucson, AZ, USA, 9-12 November.

Hill, A., Mesens, N., Steemans, M., Xu, J.J. and Aleo, M.D. (2012): Comparisons between in vitro whole cell imaging and in vivo zebrafish-based approaches for identifying potential human hepatotoxicants earlier in pharmaceutical development. Drug Metab. Rev., 44, 127-140.

Hill, A. (2011): Hepatotoxicity testing in larval zebrafish. In Zebrafish: Methods for Assessing Drug Safety and Toxicity. McGrath P (eds), pp. 89-102, Wiley-Blackwell Press, West Sussex, UK.

Hill, A.J., Teraoka, H., Heideman, W. and Peterson, R.E. (2005): Zebrafish as a model vertebrate for investigating chemical toxicity. Toxicol. Sci., 86, 6-19.

Hoofnagle, J.H., Carithers, R.L.Jr., Shapiro, C. and Ascher, N. (1995): Fulminant hepatic failure: summary of a workshop.
Hepatology, 21, 240-252.

Howe, K., Clark, M.D., Torroja, C.F., Torrance, J., Berthelot, C., Muffato, M., et al. (2013): The zebrafish reference genome sequence and its relationship to the human genome. Nature, 496, 498-503.

Hustey, G.K. and Duffull, S.B. (1999): Acute quetiapine poisoning. J. Emerg. Med., 17, 995-997.

Jollow, D.J., Mitch, J.R. and Potter, W.Z. (1973): Acetaminopheninduced hepatic necrosis. II. Role of covalent binding in vivo. J Pharmacol. Exp. Ther., 187, 195-202.

Jones, H.S., Panter, G.H., Hutchinson, T.H. and Chipman, J.K. (2010): Oxidative and conjugative xenobiotic metabolism in zebrafish larvae in vivo. Zebrafish, 7, 23-30.

Khashab, M., Tector, A.J. and Kwo, P.Y. (2007): Epidemiology of acute liver failure. Curr. Gastroenterol. Rep., 9, 66-73.

Lacy, C., Armstrong, L., Goldman, M. and Lance, L. (2001): Quetiapine. In Drug Information Handbook. 9th edition. Hudson, OH: Lexi-comp Inc, 1046-1047.

Lee, W.M. (2003): Drug-induced hepatotoxicity. N. Engl. J. Med., 349, 474-485.

MacDonald, J.S. and Robertson, R.T. (2009): Toxicity testing in the 21 st century: a view from the pharmaceutical industry. Toxicol. Sci., 110, 40-46.

McConville, B.J., Arvanitis, L.A., Thyrum, P.T., Yeh, C., Wilkinson, L.A., Chaney, R.O., et al. (2000): Pharmacokinetics, tolerability, and clinical effectiveness of quetiapine fumarate: an openlabel trial in adolescents with psychotic disorders. J. Clin. Psychiatry, 61, 252-260.

McGrath, P. and Li, C.Q. (2008): Zebrafish: a predictive model for assessing drug-induced toxicity. Drug Discov. Today, 13, $394-$ 401.

Mesens, N., Crawford, A.D., Menke, A., Hung, P.D., van Goethem, F., Nuyts, R., Hansen, E., Wolterbeek, A., van Gompel, J., De Witte, P. and Esguerra, C.V. (2015): Are zebrafish larvae suitable for assessing the hepatotoxicity potential of drug candidates? J. Appl. Toxicol., 35, 1017-1029.

Milan, D.J., Peterson, T.A., Ruskin, J.N., Peterson, R.T. and MacRae, C.A. (2003): Drugs that induce repolarization abnormalities cause bradycardia in zebrafish. Circulation, 107, 13551358.

Mutairi, F.A., Dwivedi, G. and Ameel, T.A. (2012): Fulminant hepatic failure in association with quetiapine: a case report. J. Med. Case Rep., 6, 418.

New Drug Application and Biological License Application Efficacy Supplements Approved (2004): U.S. Food and Drug Administration, Center for Drug Evaluation and Research. www.fda. gov/cder.

Norris, W., Paredes, A.H. and Lewis, J.H. (2008): Drug-induced liver injury in 2007. Curr. Opin. Gastroenterol., 24, 287-297.

O‘Brien, P.J., Irwin, W., Diaz, D., Howard-Cofield, E., Krejsa, C.M., Slaughter, M.R., et al. (2006): High concordance of druginduced human hepatotoxicity with in vitro cytotoxicity measured in a novel cell-based model using high content screening. Arch. Toxicol., 80, 580-604.

O‘Brien, P.J., Slaughter, M.R., Biagini, C., Diaz, D., Gao, B., Irwin, W., et al. (2003): Predicting drug-induced human hepatotoxicity with in vitro cytotoxicity assays. Proceedings Tox, London, UK.

Ostapowicz, G., Fontana, R.J., Schiødt, F.V., Larson, A., Davern, T.J., Han, S.H., McCashland, T.M., Shakil, A.O., Hay, J.E., Hynan, L., Crippin, J.S., Blei, A.T., Samuel, G., Reisch, J. and Lee, W.M. (2002): US Acute Liver Failure Study Group: Results of a prospective study of acute liver failure at 17 ter- 
Quetiapine fumarate induces acute hepatotoxicity

tiary care centers in the United States. Ann. Intern. Med., 137, 947-954.

Pollak, P.T. and Zbuk, K. (2000): Quetiapine fumarate overdose: clinical and pharmacokinetic lessons from extreme conditions. Clin. Pharmacol. Ther., 68, 92-97.

Reuben, A., Koch, D.G. and Lee, W.M. (2010): Drug-induced acute liver failure: results of a U.S. multicenter, prospective study. Hepatology, 52, 2065-2076.

Rizos, E., Douzenis, A., Gournellis, R., Christodoulou, C. and Lykouras, L.P. (2009): Tardive Dyskinesia in a patient treated with quetiapine. World J. Biol. Psychiatry, 10, 54-57.

Selderslaghs, I.W.T., Blust, R. and Witters, H.E. (2012): Feasibility study of the zebrafish assay as an alternativemethod to screen for developmental toxicity and embryotoxicity using a training set of 27 compounds. Reprod. Toxicol., 33, 142-154.
Seroquel package insert. (1997): Zeneca pharmaceuticals, Wilmington, DE.

Shah, R., Grover, S., Maheshwari, U., Kate, N. and Malhotra, N. (2010): Acute akathisia with quetiapine: a case report and review of the literature. Indian J. Pharmacol., 42, 416-417.

Shpaner, A., Li, W., Ankoma-Sey, V. and Botero, R.C. (2008): Druginduced liver injury: hepatotoxicity of quetiapine revisited. Eur. J. Gastroenterol. Hepatol., 20, 1106-1109.

Strachan, P.M. and Benoff, B.A. (2006): Mental status change, myoclonus, electrocardiographic changes, and acute respiratory distress syndrome induced by quetiapine overdose. Pharmacotherapy, 26, 578-582.

Zhang, C.J., Willett, C. and Fremgen, T. (2003): Zebrafish: An animal model for toxicological studies. Curr. Protoc. Toxicol., Chapter 1: Unit1.7. 